\title{
Optical Properties of Linoleic Acid Protected Gold Nanoparticles
}

\author{
Ratan Das, Siddartha S. Nath, and Ramendu Bhattacharjee \\ Department of Physics, Assam University, Silchar, Assam 788011, India \\ Correspondence should be addressed to Siddartha S. Nath, nathssrd@gmail.com
}

Received 16 April 2010; Revised 3 July 2010; Accepted 22 July 2010

Academic Editor: Quanqin Dai

Copyright (C) 2011 Ratan Das et al. This is an open access article distributed under the Creative Commons Attribution License, which permits unrestricted use, distribution, and reproduction in any medium, provided the original work is properly cited.

\begin{abstract}
Linoleic acid-protected gold nanoparticles have been synthesized through the chemical reduction of tetrachloroaurate ions by ethanol in presence of sodium linoleate. The structure of these nanoparticles is investigated using transmission electron microscopy, which shows that the Au nanoparticles are spherical in shape with a narrow size distribution which ranges from 8 to $15 \mathrm{~nm}$. Colloidal dispersion of gold nanoparticles in cyclohexane exhibits absorption bands in the ultraviolet-visible range due to surface plasmon resonance, with absorption maximum at $530 \mathrm{~nm}$. Fluorescence spectra of gold nanoparticles also show an emission peak at $610 \mathrm{~nm}$ when illuminated at $450 \mathrm{~nm}$. UV-Vis spectroscopy reveals that these nanoparticles remain stable for 10 days.
\end{abstract}

\section{Introduction}

Nanoparticles $(1-100 \mathrm{~nm})$ have been a source of intense interest due to their novel electrical, optical, physical, chemi$\mathrm{cal}$, and magnetic properties. They have significant potential for a wide range of applications such as catalysis, magnetic recording media, optoelectronic materials, magnetic fluids, composite materials, fuel cells, pigments, and sensors. Their uniqueness arises from their high ratio of surface area to volume [1-6]. The synthesis of noble metal nanoparticles with desired size/shape has enormous importance, especially in the emerging field of nanotechnology. There is presently great interest in the optical properties of gold nanoparticles due to the various applications they have in different fields. These gold colloids show bright colour due to surface plasmon resonance (SPR). This surface plasmon resonance is the transverse oscillations of the surface electrons of the particle on interaction with light of suitable wavelength. Gold colloids show an absorption band in the visible range. Here gold nanoparticles have been prepared by a simple and convenient reduction method [7], which is a promising approach for highly efficient synthesis of gold nanoparticles with a more uniform particle size. The prepared gold nanoparticles have been dispersed in cyclohexane and then examined using X-ray diffraction (XRD), Transmission Electron Microscope (TEM), UV/Vis absorption spectroscopy, and fluorescence spectroscopy (PL). The most important factor is that the gold nanoparticles prepared by this process remain stable for 10 days.

\section{Materials and Method}

Uniform gold nanoparticles can be obtained through the reduction of tetrachloroaurate ions by ethanol at temperatures between $30^{\circ} \mathrm{C}$ to $60^{\circ} \mathrm{C}$ under atmospheric conditions [7]. In this synthesis process, $25 \mathrm{~mL}$ of aqueous solution containing tetrachloroauric acid ( $1 \mathrm{~g}$ of $\left.\mathrm{HAuCl}_{4}\right), 3 \mathrm{~g}$ sodium linoleate $\left(\mathrm{C}_{18} \mathrm{H}_{32} \mathrm{ONa}\right), 20 \mathrm{~mL}$ ethanol $\left(\mathrm{C}_{2} \mathrm{H}_{5} \mathrm{OH}\right)$, and $5 \mathrm{~mL}$ linoleic acid $\left(\mathrm{C}_{18} \mathrm{H}_{32} \mathrm{O}_{2}\right)$ are added in a capped tube under agitation. The system is well capped and treated at the temperature range $30^{\circ} \mathrm{C}$ to $60^{\circ} \mathrm{C}$ for 2 hours. In the aqueous solution of tetrachloroauric acid, sodium linoleate and the mixture of linoleic acid and ethanol are combined in order: a solid phase of sodium linoleate, a liquid phase of ethanol and linoleic acid, and water ethanol solution phase containing tetrachloroaurate ions forms in the system. Ethanol in the liquid and solution phases reduces the tetrachloroaurate ions into gold nanoparticles. Along with the reduction process, among all the reactant, only the linoleic acid gets absorbed on the surface of the gold nanoparticles with the alkyl chains on the outside through which the produced nanoparticles gain hydrophobic surfaces. In this simple reduction process, 


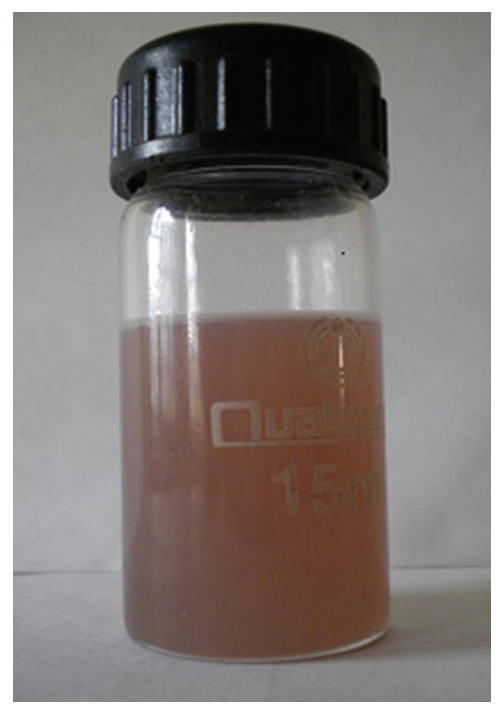

FIGURE 1: Pale reddish colour of the gold nanoparticles dispersed in cyclohexane.

the role of linoleic acid is to protect the gold nanoparticle from agglomeration, making a layer over it with its alkyl chains on the outside giving a hydrophilic surroundings to the nanoparticles. On changing the concentration of the electrolyte, it is found that the colour becomes reddish on adding linoleic acid at the same proportion. This reddish colour of prepared nanoparticles indicates the conversion of tetrachloroaurate ions into gold nanoparticles.

The produced nanoparticles having reddish brown in colour actually, collected at the bottom of vessel after cooling to room temperature, are then dispersed in cyclohexane to form a homogenous colloidal solution of gold nanoparticles. The colour of the colloidal solution of gold nanoparticles becomes pale reddish due to dispersion in cyclohexane as shown in Figure 1.

The structure of prepared gold nanoparticles has been investigated by Bruker AXS model X-ray diffractometer. Size and shape of the gold nanoparticles has been obtained from TEM photographs which was performed on a JEM 1000C X II model instrument. The absorption spectra of the gold nanoparticles was taken on a Perkin Elmer Lambda 351.24 model whereas fluorescence spectra was performed on Hitachi; F-2500 model.

\section{Results and Discussion}

3.1. XRD Analysis. The $\mathrm{XRD}$ patterns of the sample prepared by the present reduction method are shown in Figure 2. The crystalline nature of the as-synthesized product is demonstrated in the XRD measurements. The diffraction peaks at 45.3 degrees and 64.5 degrees match with those of a facecentred cubic ( $\mathrm{fcc}$ ) Au as in JCPDS correspond to the planes of (200) and (220), respectively. From this study, average particle size is estimated by using Debye-Scherrer formula $[8,9]$. Here the instrumental broadening at 45.3 degrees and 64.5 degrees are 0.00632 and 0.00912 . Considering

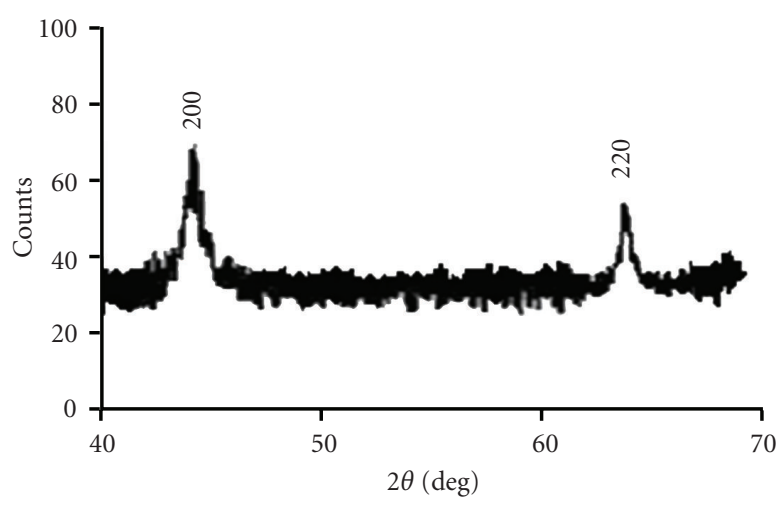

FIGURE 2: XRD pattern of gold nanoparticles.

the instrumental broadening, the average particle size is calculated to be around $(11 \pm 2) \mathrm{nm}$ with $14 \%$ experimental error.

3.2. TEM Analysis. Samples for TEM studies are prepared by placing a drop of the gold colloidal solution on TEM carboncoated copper grid. The films on the TEM grids are allowed to dry for a few hours after removing the extra solution using blotting paper. TEM micrograph of the prepared colloidal solution of gold nanoparticles is shown in Figure 3(a) with a graphic of statistical size distribution of particles in Figure 3(b). The Au nanoparticles (black portion) are spherical in shape with smooth surface morphology. Though the size range of the nanoparticle lie between 8 and $15 \mathrm{~nm}$, about $80 \%$ of the nanoparticles were found to have size approximately $12 \mathrm{~nm}$. After 10 days, it is found that the reddish colour of the colloidal solution starts to deteriorate slowly and the particles starts to agglomerate with time. So, after 10 days particles remain no longer stable.

3.3. UV/Vis Spectroscopy Analysis. Noble metal nanoparticles ( $\mathrm{Au}, \mathrm{Ag}, \mathrm{P}$, etc.) show a strong absorption band in the visible range due to surface plasmon resonance. Surface plasmon resonance is the coherent motion of free electrons in the conduction band caused by interaction with an electromagnetic field. The electric field of an incoming light wave induces a polarization of electrons with respect to the heavier ionic core of the nanoparticles. This induces a dipolar oscillation of all the free electrons with the same phase. When the frequency of the light wave become resonant with the electron motion, a strong absorption occurs which is the origin of observed colour of colloids. Width of the absorption band and wavelength corresponding to the surface plasmon resonance peak strongly depend on size, shape, dielectric constant of nanoparticle, as well as on the surrounding medium. For small metal particles (diameter $<20 \mathrm{~nm}$ ), absorption spectra significantly depend only on the dipole oscillation $[5,6,10-13]$. This reduction method by ethanol produces spherical gold nanoparticles around $12 \mathrm{~nm}$. The UV/Vis absorption spectra of the gold nanoparticles dispersed in cyclohexane is shown in Figure 4. The absorption (SPR) peak is obtained in the visible range at $530 \mathrm{~nm}$ which matches well with others result. Moreover, 


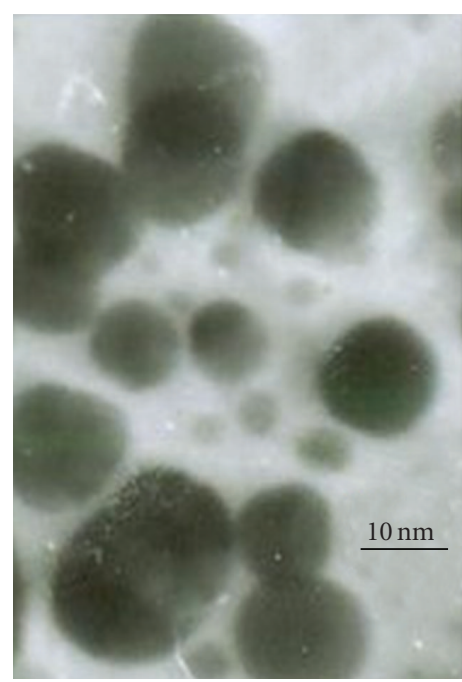

(a)

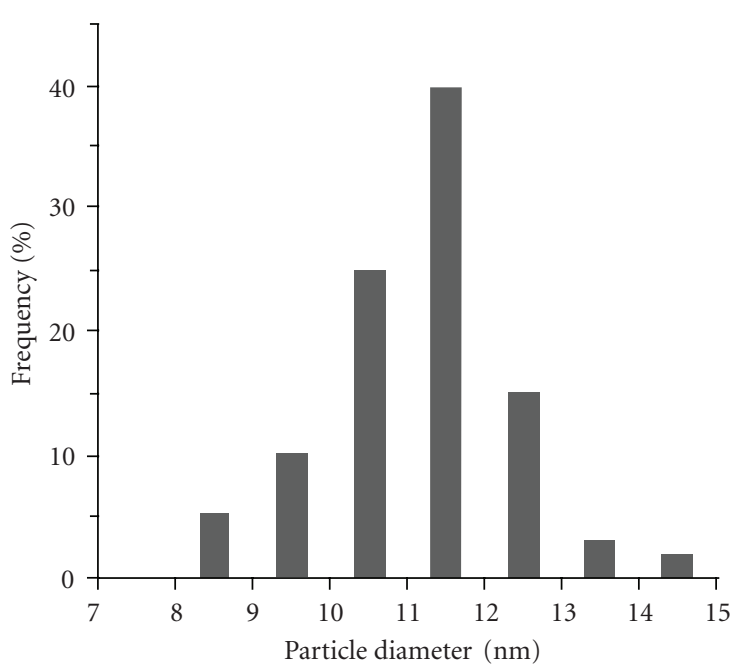

(b)

FIgURE 3: (a) TEM image of gold nanoparticles. (b) Graphic for particle size distribution.

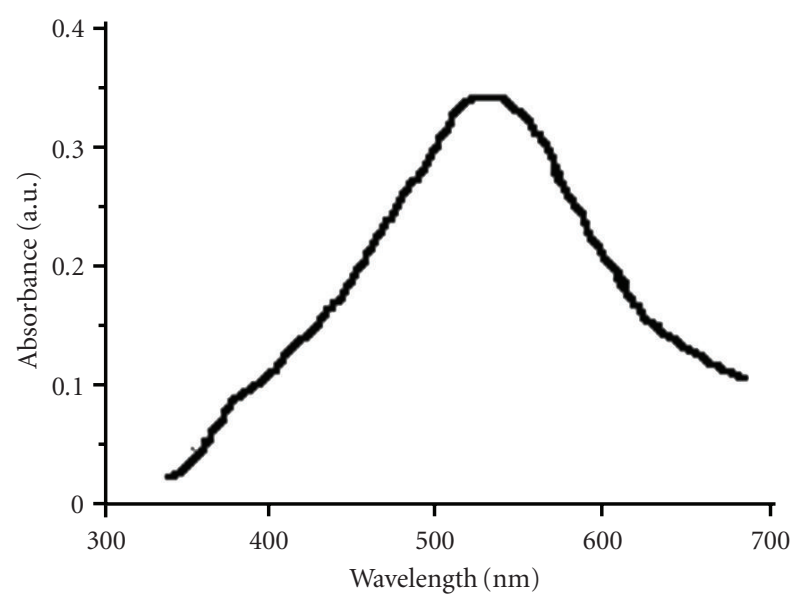

Figure 4: UV-Vis spectra of gold nanoparticles.

as absorption peak and width remains the same for 10 days, so it indicates that nanoparticles remain stable for 10 days without any agglomeration.

3.4. Fluorescence Spectroscopy. In gold nanoparticles, 5d valence and $6 \mathrm{sp}$-conduction electrons play the role for fluorescence spectra [12-18]. It has been reported by Liao et al. [14] and Varnavski et al. [18] that $15 \mathrm{~nm}$ particles produce fluorescence. Eichelbaum et al. [16] reported that $10 \mathrm{~nm}$ gold nanoparticles show photoluminescence. The reason for photoluminescence is attributed to the interband transition from sp-conduction band above the Fermi level to the dband below. The radiative recombination of electron hole pairs between this d-band and sp-conduction band produces emission [12-18], which occurs at $610 \mathrm{~nm}$ for linoleic acid protected gold nanoparticles $(8-15 \mathrm{~nm})$, when excited with $450 \mathrm{~nm}$ of optical source (Xenon lamp-150 watt). Moreover, the absorbed linoleic acid on gold nanoparticles

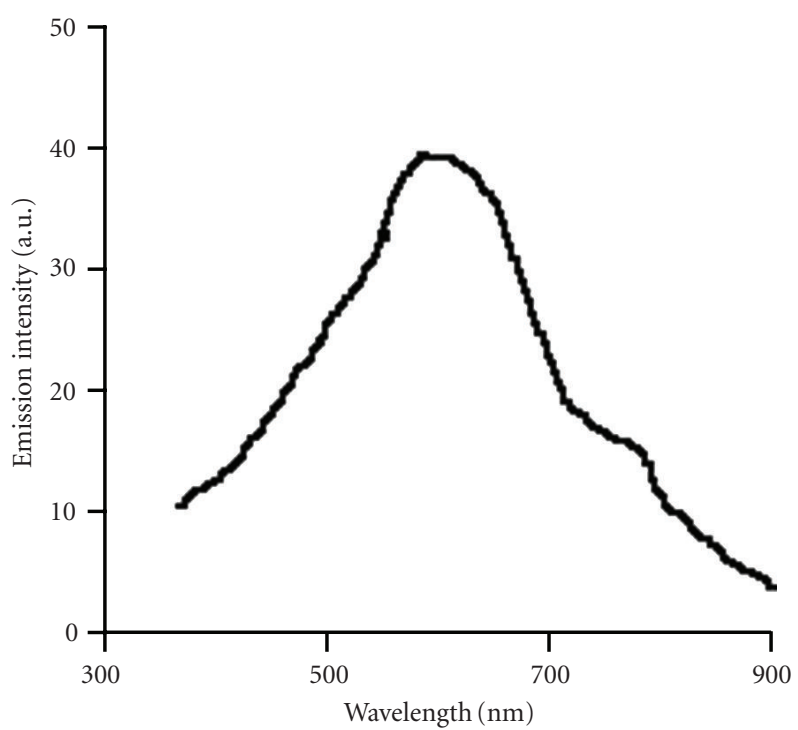

FIGURE 5: Fluorescence spectra of gold nanoparticles.

surface further enhances the intensity of emission [14-17]. Fluorescence spectra for gold nanoparticle are shown in Figure 5.

\section{Summary}

We have prepared gold nanoparticles through the reduction of tetrachloroaurate ions by ethanol, which is dispersed in cyclohexane. Here, linoleic acid acts as a stabilizer. XRD analysis, UV/Vis spectra, and TEM micrograph reveal that the prepared nanoparticles are spherical in shape with an average size of $12 \mathrm{~nm}$ which remain stable for 10 days. SPR peak is observed at $530 \mathrm{~nm}$ whereas fluorescence peak is obtained at $610 \mathrm{~nm}$. 


\section{Acknowledgments}

The authors thank to Dr D. K. Avasthi (Scientist H) Material Science, IUAC, New Delhi, India, Dr B. DKHR (S.O.) NEHU, Shillong, India, Dr. S. Sharma (S.O.), IIT, Guwahati, Assam, India and Dr. Sudip Choudhury, lecturer, G. C. College, Silchar, Assam, India for their suggestions and assistance during the work.

\section{References}

[1] G. Cao, Nanostructures and Nanomaterials, Imperial College Press, London, UK, 2004.

[2] M. A. Reed and T. Lee, Molecular Nanoelectronics, American Scientific, Stevenson Ranch, Calif, USA, 2003.

[3] C. P. Poole and F. J. Owens, Introduction to Nanotechnology, Wiley Interscience, New York, NY, USA, 2003.

[4] A. Taleb, C. Petit, and M. P. Pileni, "Optical properties of selfassembled 2D and 3D superlattices of silver nanoparticles," Journal of Physical Chemistry B, vol. 102, no. 12, pp. 2214 2220, 1998.

[5] M. A. Noginov, G. Zhu, M. Bahoura et al., "The effect of gain and absorption on surface plasmons in metal nanoparticles," Applied Physics B, vol. 86, no. 3, pp. 455-460, 2006.

[6] C. Sönnichsen, T. Franzl, T. Wilk, G. Von Plessen, and J. Feldmann, "Plasmon resonances in large noble-metal clusters," New Journal of Physics, vol. 4, pp. 93.1-93.8, 2002.

[7] X. Wang, J. Zhuang, Q. Peng, and Y. Li, "A general strategy for nanocrystal synthesis," Nature, vol. 437, no. 7055, pp. 121-124, 2005.

[8] D. Mohanta, S. S. Nath, N. C. Mishra, and A. Choudhury, "Irradiation induced grain growth and surface emission enhancement of chemically tailored $\mathrm{ZnS}: \mathrm{Mn} / \mathrm{PVOH}$ nanoparticles by $\mathrm{Cl}^{+9}$ ion impact," Bulletin of Materials Science, vol. 26, no. 3, pp. 289-294, 2003.

[9] S. S. Nath, D. Chakdar, G. Gope et al., "Green luminescence of $\mathrm{ZnS}$ and $\mathrm{ZnS}$ :Cu quantum dots embedded in zeolite matrix," Journal of Applied Physics, vol. 105, no. 9, Article ID 094305, 2009.

[10] R. Das, S. S. Nath, D. Chakdar, G. Gope, and R. Bhattacharjee, "Preparation of silver nanoparticles and their characterization," Journal of Nanotechnology, vol. 5, pp. 1-6, 2009.

[11] R. Das, S. S. Nath, D. Chakdar, G. Gope, and R. Bhattacharjee, "Synthesis of silver nanoparticles and their optical properties," Journal of Experimental Nanoscience, vol. 5, no. 4, pp. 357-362, 2010.

[12] M. P. Pileni, "Optical properties of nanosized particles dispersed in colloidal solutions or arranged in $2 \mathrm{D}$ or $3 \mathrm{D}$ superlattices," New Journal of Chemistry, vol. 22, no. 7, pp. 693-702, 1998.

[13] S. Link and M. A. El-Sayed, "Shape and size dependence of radiative, non-radiative and photothermal properties of gold nanocrystals," International Reviews in Physical Chemistry, vol. 19, no. 3, pp. 409-453, 2000.

[14] H. Liao, W. Wen, and G. K.L. Wong, "Photoluminescence from $\mathrm{Au}$ nanoparticles embedded in Au:oxide composite films," Journal of the Optical Society of America B, vol. 23, no. 12, pp. 2518-2521, 2006.

[15] J. Zhang, J. Malicka, I. Gryczynski, and J. R. Lakowicz, "Oligonucleotide-displaced organic monolayer-protected silver nanoparticles and enhanced luminescence of their salted aggregates," Analytical Biochemistry, vol. 330, no. 1, pp. 81-86, 2004.
[16] M. Eichelbaum, B. E. Schmidt, H. Ibrahim, and K. Rademann, "Three-photon-induced luminescence of gold nanoparticles embedded in and located on the surface of glassy nanolayers," Nanotechnology, vol. 18, no. 35, Article ID 355702, 8 pages, 2007.

[17] J. Xu, X. Han, H. Liu, and Y. Hu, "Synthesis and optical properties of silver nanoparticles stabilized by gemini surfactant," Colloids and Surfaces A, vol. 273, no. 1-3, pp. 179-183, 2006.

[18] O. Varnavski, R. G. Ispasoiu, L. Balogh, D. Tomalia, and T. Goodson III, "Ultrafast time-resolved photoluminescence from novel metal-dendrimer nanocomposites," Journal of Chemical Physics, vol. 114, no. 5, pp. 1962-1965, 2001. 

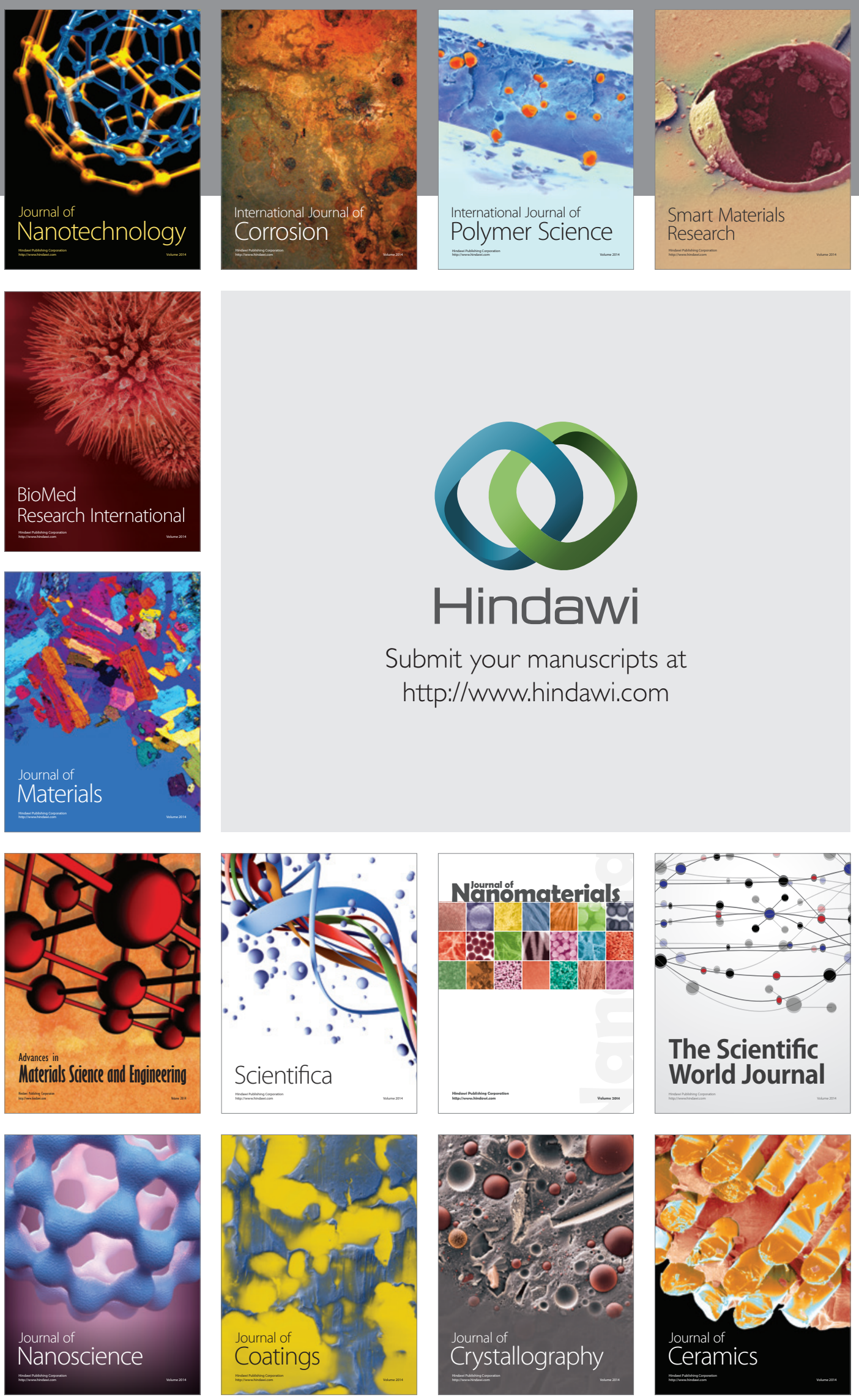

The Scientific World Journal

Submit your manuscripts at

http://www.hindawi.com

\section{World Journal}

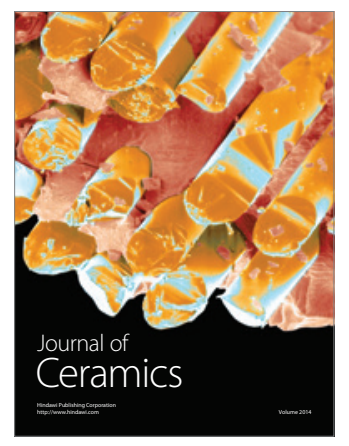

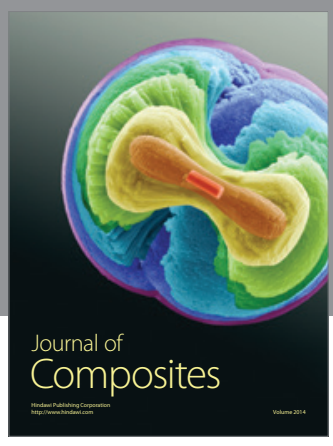
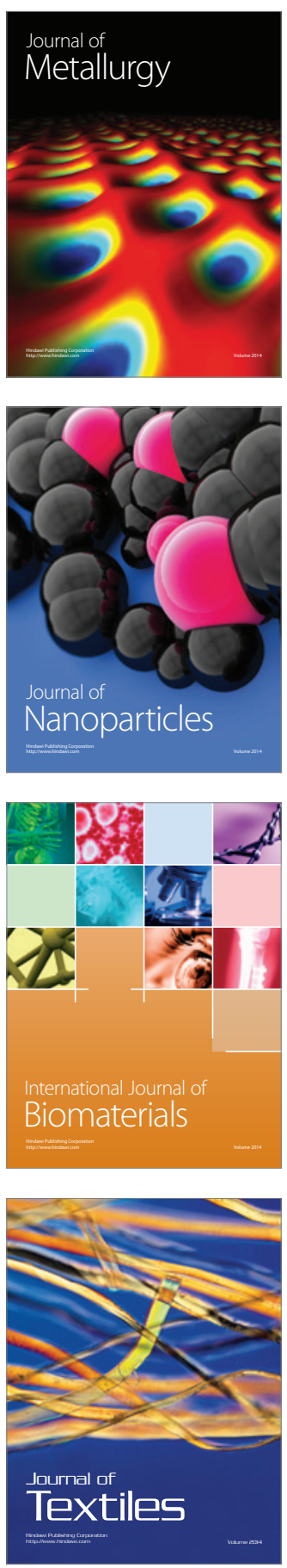